\title{
Carbon-ion radiotherapy boost with standard dose proton radiation for incomplete-resected high-grade glioma: a phase 1 study
}

\author{
Xianxin Qiu ${ }^{1,2,3,4 \#}$, Jing Gao ${ }^{1,2,4 \#}$, Jing Yang ${ }^{1,2,4}$, Jiyi Hu ${ }^{1,2,4}$, Weixu Hu ${ }^{1,2,4}$, Qingting Huang ${ }^{1,2,4}$, Lin Kong ${ }^{2,3,4}$, \\ Jiade J. Lu ${ }^{1,2,4}$
}

${ }^{1}$ Department of Radiation Oncology, Shanghai Proton and Heavy Ion Center, Shanghai, China; ${ }^{2}$ Shanghai Engineering Research Center of Proton and Heavy Ion Radiation Therapy, Shanghai, China; ${ }^{3}$ Department of Radiation Oncology, Shanghai Proton and Heavy Ion Center, Fudan University Cancer Center, Shanghai, China; ${ }^{4}$ Shanghai Key Laboratory of Radiation Oncology (20dz2261000), Shanghai, China

Contributions: (I) Conception and design: JJ Lu, L Kong; (II) Administrative support: JJ Lu; (III) Provision of study materials or patients: X Qiu, J Gao, J Yang, W Hu, Q Huang, L Kong, JJ Lu; (IV) Collection and assembly of data: X Qiu, J Gao, J Yang, W Hu, Q Huang; (V) Data analysis and interpretation: X Qiu, J Gao, J Hu; (VI) Manuscript writing: All authors; (VII) Final approval of manuscript: All authors.

\#These authors contributed equally to this work.

Correspondence to: Lin Kong, MD. Shanghai Proton and Heavy Ion Center, Fudan University Cancer Center, 4365 Kangxin Road, Pudong, Shanghai, China. Email: lin.kong@sphic.org.cn; Jiade J. Lu, MD, MBA. Shanghai Proton and Heavy Ion Center, 4365 Kangxin Road, Pudong, Shanghai, China. Email: jiade.lu@sphic.org.cn.

Background: To investigate the maximal tolerated dose (MTD) of a carbon-ion radiotherapy (CIRT) boost prior to standard dose proton radiotherapy (PRT) for newly diagnosed glioblastoma (GBM) and anaplastic astrocytoma (AA) patients with residual lesion after resection.

Methods: In total, 18 patients with high-grade glioma (HGG) (16 with GBM and 2 with AA) were enrolled in a prospective $3 \times 3$ design phase 1 trial. We investigated four dose-levels of CIRT boost [9 (starting level), 12,15 , and 18 Gy relative biological effectiveness (RBE)] delivered in three equal fractions prior to the standard dose PRT (60 Gy RBE in 30 fractions). Concurrent temozolomide (TMZ) was not provided during the CIRT boost but was initiated on the first day of PRT. Acute and late toxicities were scored based on the Common Terminology Criteria for Adverse Events (CTCAE, v 4.03). Dose-limiting toxicities (DLTs) were defined as radiation-induced severe toxicities ( $\geq$ grade 3 ).

Results: With a median follow-up of 17.9 months, no severe ( $\geq$ grade 3 ) acute or late toxicities were observed in patients treated with the first three dose levels (CIRT boost doses of 9, 12, 15 Gy RBE). Severe late toxicity (grade 3 radiation necrosis) was observed in the first patient treated with the 18 Gy RBE CIRT boost level. Therefore, this trial was terminated and the MTD of the induction CIRT boost was determined at $15 \mathrm{~Gy} \mathrm{RBE}$ in 3 fractions. At the time of this analysis, both patients with AA were alive without disease progression. The progression-free survival (PFS) and overall survival (OS) for GBM at 12 months were $50.6 \%$ and $78.6 \%$, respectively.

Conclusions: Particle beam radiotherapy consisting of a CIRT boost of 15 Gy RBE (in 3 fractions) following standard dose PRT (60 Gy RBE in 30 fractions), and used in conjunction with TMZ, is safe and potentially effective for patients with HGG.

Keywords: Glioblastoma (GBM); anaplastic astrocytoma (AA); carbon-ion radiotherapy (CIRT); proton radiotherapy (PRT); temozolomide (TMZ)

Submitted Sep 03, 2020. Accepted for publication Jan 08, 2021.

doi: $10.21037 /$ atm-20-7750

View this article at: https://dx.doi.org/10.21037/atm-20-7750 


\section{Introduction}

High-grade glioma (HGG) is the most frequently diagnosed and aggressive primary brain malignancy in adults (1). Currently, surgical resection followed by adjuvant radiotherapy in conjunction with temozolomide (TMZ) chemotherapy is the standard treatment. The median survival time (MST) of newly diagnosed patients with anaplastic astrocytoma (AA) and glioblastoma (GBM) is approximately 36 and 15 months, respectively $(2,3)$. Significant efforts of developing novel therapeutic agents for GBM, such as agents targeting Notch pathway, vascular endothelial growth factor (VEGF) signaling, plateletderived growth factor (PDGF) signaling, have been made but all failed to further improve the survival of GBM patients in recent years (4). Progression or recurrence occurring close to the radiation field is nearly universal, despite the aggressive treatment (2). Attempts with more aggressive radiation therapy strategies or technologies, such as the addition of stereotactic radiosurgery (SRS), altered fractionation, and dose escalation have failed to demonstrate significant survival benefits (5-8). Clearly, more effective radiation therapy approaches are needed to further improve disease control for HGG, and in particular, GBM.

One of the core challenges of radiation therapy for treating GBM, either definitively or adjuvantly, is employing higher doses to the disease volume, while at the same time, exposing normal critical brain tissue to the lowest possible dose of radiation. Particle (e.g., proton and carbon-ion) beam, as the most advanced radiation technique, deposits the majority of the energy to a highly localized region (i.e., the Bragg peak), thereby enabling more precise dose distribution over photon beams. Numerous studies have reported that particle beam radiation therapy (PBRT), and proton beam therapy in particular, provides improved dose distribution in the treatment of glioma, suggesting theoretically improved efficacy and side-effect profiles (9-11). Furthermore, carbon-ion beam radiation has higher linear energy transfer (LET) compared to photon and proton beams, and can therefore inflict greater damage via direct deoxyribonucleic acid (DNA) double-strand breaks and tumor cell-killing (12). Results from numerous studies have demonstrated superior outcomes for several types malignancies treated with carbon-ion radiotherapy (CIRT) (13-15).

At the Shanghai Proton and Heavy Ion Center (SPHIC), proton radiation therapy (PRT) to the standard dose has been used for patients with HGG. To investigate the safety and efficacy of the addition of a CIRT boost to the gross residual HGG delivered prior to the standard PRT, a randomized phase I/III clinical trial has been initiated (China Clinical Trials Registry ID: ChiCTR-OID-17013702) (16). The purpose of this study is to report the results of phase I of the trial, which was designed to determine the maximal tolerated dose (MTD) of a CIRT boost in the combined radiation modality. We present the following article in accordance with the TREND reporting checklist (available at https://atm.amegroups.com/article/view/10.21037/atm$20-7750 / \mathrm{rc})$.

\section{Methods}

\section{Patients (eligibility criteria)}

Patients with histologically diagnosed, unifocal, supratentorial primary GBM or AA and indications for radiation therapy in conjunction with TMZ chemotherapy were eligible for the trial. Additional inclusion criteria were as follows: age $\geq 18$ years; Karnofsky performance score (KPS) $\geq 60$; and gross tumor (for patients who underwent biopsy) or residual tumor (for patients who underwent partial resection) with the largest dimension up to $5 \mathrm{~cm}$ assessed by postoperative multimodal brain imaging studies.

Patients were excluded from this trial if they met any of the following criteria: (I) previous exposure to brain radiation for any condition; (II) previous malignant tumor of any type requiring cytotoxic therapy within 5 years prior to enrollment; (III) no residual lesion detected by postoperative multimodal brain imaging studies; (IV) a time interval $>8$ weeks between surgery and initiation of radiotherapy; (V) pregnant or lactating (female); (VI) severe pulmonary hypertension, cardiovascular disease, peripheral vascular disease, severe chronic heart disease, and other complications that may interfere with radiotherapy. The study was conducted in accordance with the Declaration of Helsinki (as revised in 2013). The study was approved by the ethics committee at Shanghai Proton and Heavy Ion Center (approval number: 1611-12-031704A). Written informed consent was obtained from all patients.

All patients were required to be present in the multidisciplinary tumor clinic for their diagnosis, indication, and eligibility for the PBRT protocol prior to registration at the SPHIC. Required pretreatment evaluation included a complete history and physical examination, full blood count, serum electrolytes, liver and renal function tests, and magnetic resonance imaging (MRI) of the brain. 


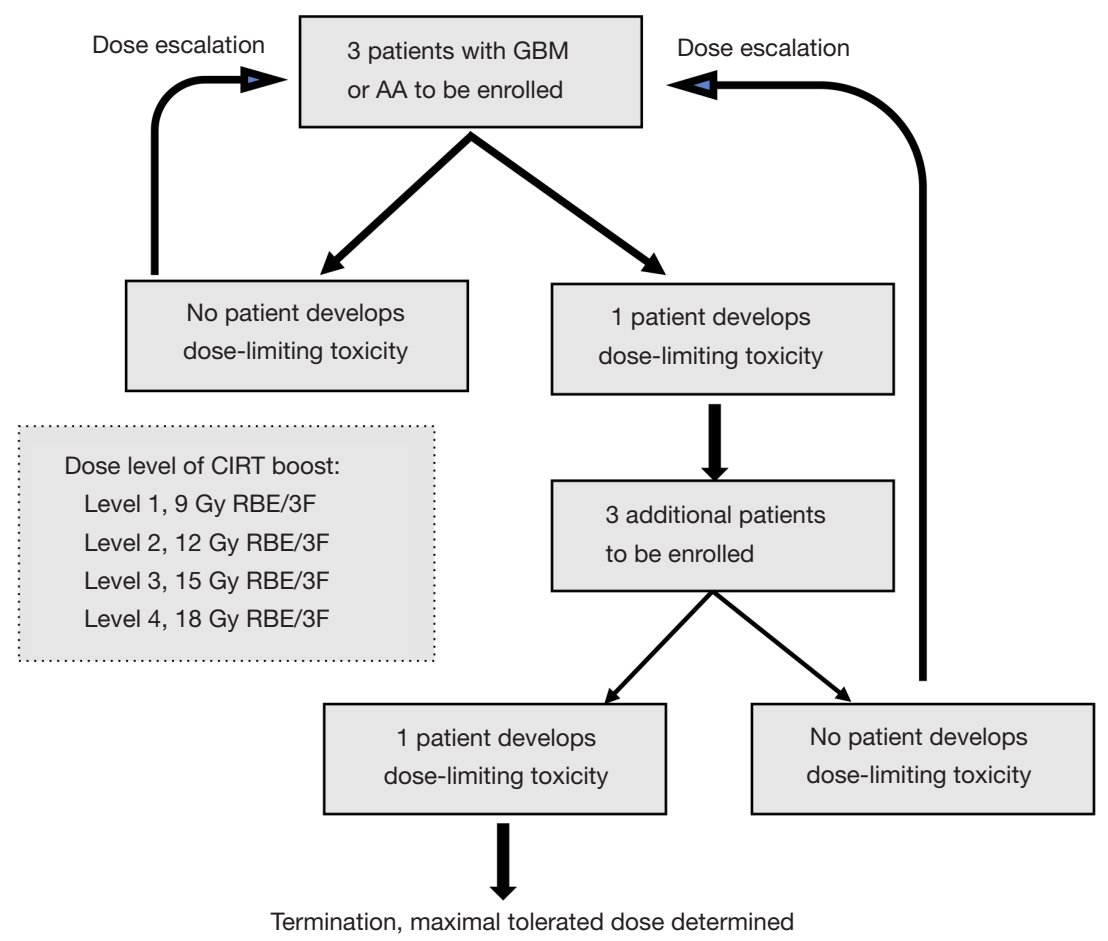

Figure 1 The flowchart of this phase I trial. AA, anaplastic astrocytoma; CIRT, carbon-ion radiotherapy; GBM, glioblastoma; RBE, relative biological effectiveness.

\section{Study designs}

The primary objective of this phase I trial was to determine the MTD of a CIRT boost in addition to the standard dose PRT [60 Gy relative biological effectiveness (RBE) in 30 fractions]. Four dose-levels of CIRT boost [9 (starting level), 12, 15, and 18 Gy RBE] delivered in three equal fractions prior to PRT were investigated. The assessment for doselimiting toxicities (DLTs) was performed from the initiation of radiotherapy according to the Common Terminology Criteria for Adverse Events (CTCAE), v 4.03. DLTs were defined as radiation-induced severe toxicities ( $\geq$ grade 3 ).

The study used a $3 \times 3$ design, and the CIRT boost was initiated at 9 Gy RBE in 3 fractions. In the absence of any DLTs, we continued to the next dose escalation level of the CIRT boost with three patients. An observation interval of at least 6 months was required prior to the initiation of the next dose level. Three more patients would be required to a dosing group if any DLT was observed in one case. If two or more cases of DLTs were observed in the three patients treated at any level, the study was to be terminated and the CIRT boost dose used in the preceding dose level was chosen as the MTD. During the 6-month observation period, additional patients, if available, were accrued and treated according to the prior dose level. Figure 1 summarizes the flow chart of this phase I trial design.

\section{Radiotherapy planning}

All patients with brain tumors were immobilized in the supine position using VacLock ${ }^{\circledR}$ and individualized thermoplastic masks. Computed tomography (CT) for simulation from the vertex to the inferior margin of the second cervical vertebral body was performed at $1.5-\mathrm{mm}$ slice thickness.

Multimodal brain imaging techniques, including perfusion weighted imaging (PWI), diffusion tensor imaging (DTI), magnetic resonance spectroscopy (MRS), blood oxygenation level-dependent imaging (BOLD), and C-methionine/fluoro-ethyl-tyrosine (MET/FET) positron emission tomography (PET) scans can incorporate comprehensive information about the tumor burden and critical brain structure to improve the radiation plan for GBM. These techniques were employed to delineate the gross tumor volume (GTV) for the CIRT boost (GTVboost), in addition to T1-weighted contrast enhanced and fluid attenuated inversion recovery (FLAIR) MRI. 
The clinical target volume (CTV) for high risk (CTV-hr) consisted of GTV-boost and the surgical bed plus $5 \mathrm{~mm}$ expansion; the CTV for low risk (CTV-lr) consisted of GTV-boost and the surgical cavity with a $15 \mathrm{~mm}$ expansion plus the peritumoral edema area. An additional 3-5 mm margin was supplied to the CTVs to create the planning target volume (PTV) for uncertainty with regard to dose distribution and potential daily setup errors.

The CIRT boost was performed as an induction method to cover the GTV-boost prior to PRT. The prescribed PRT doses to CTV-hr and CTV-1r were 60 Gy RBE and 50 Gy $\mathrm{RBE}$, respectively, in 30 fractions using the simultaneously integrated boost (SIB) technique. The dose constraints of critical organs at risk (OARs) were based on the tolerance dose (TD) $)_{5 / 5}$ described by Emami et al. (17).

Both the CIRT boost and PRT were planned using the Syngo ${ }^{\circledR}$ treatment planning system, and delivered with pencil beam scanning (PBS) technology using the IONTRIS ${ }^{\circledR}$ particle therapy system (Siemens, Erlangen, Germany). All patients were treated on in-patient basis. Orthogonal X-ray verification was conducted for position using bony landmarks as references prior to each delivery. The anatomical changes of the lesion were evaluated by weekly verification CT scans, with additional MRI ordered if the CT scan indicated major change(s), which may alter the dose coverage.

\section{Chemotherapy}

TMZ was provided concurrently as an adjuvant treatment to all patients aged $\leq 65$ years regardless of the methylation status of the $\mathrm{O}[6]-$ methylguanine-DNA methyltransferase (MGMT) promoter, and to patients older than 65 years with methylation of the MGMT promoter. Concurrent oral TMZ ( $75 \mathrm{mg} / \mathrm{m}^{2}, 7$ days/week) was not provided during the CIRT boost, but was initiated on the first day of PRT. Adjuvant TMZ was administered from 1-month after the completion of PRT for at least six cycles at $150-200 \mathrm{mg} / \mathrm{m}^{2}$ and for 5 days during every 28-day cycle.

\section{Follow-up}

All patients were examined daily during PBRT, and were required to be followed-up regularly according to the SPHIC institutional follow-up protocol after the completion of PBRT. The first and second follow-up visits were scheduled at 1 and 3 months after discharge, respectively. Further follow-up sessions were planned every $2-3$ months in the first 3 years, every $4-6$ months in the following 2 years, and annually thereafter. A complete history and physical examination with a focus on neurological studies, brain MRI, and routine lab tests were provided at each follow-up.

\section{Assessments for toxicities and treatment response}

Toxicities that occurred during or within 3 months after the initiation of PBRT were defined as acute toxicities, and those that developed after 3 months from or persisted for at least 3 months after the initiation of PBRT were defined as late toxicities. Both acute and late toxicities were scored based on the CTCAE, v4.03.

The target lesions were evaluated based on MRI findings using the Response Assessment in Neuro-oncology (RANO) Criteria (18) with interpretation modifications (19), which are summarized as follows:

(I) Complete response (CR): complete disappearance of all tumors on consecutive MRI scans sustained for at least 1 month without the use of steroids;

(II) Partial response (PR): an observed $\geq 50 \%$ decrease in the area of contrast enhancement on consecutive MRI sustained for at least 1 month. Doses of steroids must be stable or decreasing, and the patient must be neurologically stable;

(III) Progressive disease (PD): an observed $\geq 25 \%$ increase in the area of contrast enhancement or any new tumor on MRI;

(IV) Stable disease (SD): all other situations.

\section{Statistics}

Overall survival (OS) time was defined as the duration between pathological diagnosis and the date of death. Progression-free survival (PFS) time was calculated as the duration between the time of pathological diagnosis and the date of disease progression, diagnosed either clinically or histologically. The OS and PFS rates were calculated using the Kaplan-Meier method. All statistical analyses were performed using the IBM ${ }^{\circledR}$ SPSS statistics software (version 26).

\section{Results}

\section{Patient population}

Between May 2017 and October 2019, a total of 18 patients (11 males and 7 females) were enrolled in this study. Two patients $(11.1 \%)$ were diagnosed with AA [World Health Organization (WHO) grade III], while the remaining 
Table 1 Patient characteristics

\begin{tabular}{|c|c|}
\hline Characteristics & No. of patients \\
\hline Whole series (n) & 18 \\
\hline \multicolumn{2}{|l|}{ Gender } \\
\hline Male & 11 \\
\hline Female & 7 \\
\hline \multicolumn{2}{|l|}{ Age (years) } \\
\hline Median [range] & 56.5 [48-69] \\
\hline$<50$ & 4 \\
\hline$\geq 50$ & 14 \\
\hline \multicolumn{2}{|c|}{ Completeness of resection } \\
\hline Partial/biopsy & 1 \\
\hline Subtotal & 17 \\
\hline \multicolumn{2}{|l|}{ KPS } \\
\hline$>80$ & 15 \\
\hline$\leq 80$ & 3 \\
\hline \multicolumn{2}{|c|}{ Histology grade (WHO grade) } \\
\hline Grade IV & 16 \\
\hline Grade III & 2 \\
\hline \multicolumn{2}{|l|}{ IDH mutation } \\
\hline Wild type & 14 \\
\hline Mutant & 4 \\
\hline \multicolumn{2}{|l|}{ MGMT promoter } \\
\hline Methylated & 4 \\
\hline Un-methylated & 10 \\
\hline N/A & 4 \\
\hline
\end{tabular}

KPS, karnofsky performance score; WHO, World Health Organization; IDH, isocitrate dehydrogenase; MGMT, O[6]methylguanine-DNA methyltransferase; N/A, not available.
16 patients $(88.9 \%)$ were diagnosed with GBM (WHO grade IV). All patients received concurrent and adjuvant oral TMZ according to the Stupp protocol (2) and based on their age or the methylation status of the MGMT promoter. The baseline characteristics of the patients, as well as the histological and molecular findings are detailed in Table 1. The dose level and dose volume prescriptions are shown in Table 2.

\section{Toxicities and MTD}

The observed acute and late radiation-related toxicities are listed in Table 3. No severe (i.e., grade 3 or above) acute or late toxicity was observed in patients treated with the first three dose levels (i.e., CIRT boost doses of 9, 12, 15 Gy RBE). However, severe late toxicity (grade 3 radiation necrosis) was observed in the first patient treated with the 18 Gy RBE CIRT boost level at 4 months after the completion of PBRT. Dexamethasone and mannitol were administered to prevent worsening symptoms of the patient. Therefore, this trial was terminated and the MTD of induction CIRT boost was determined at 15 Gy RBE in 3 fractions.

\section{Disease control and survival outcomes}

The median follow-up time of the entire cohort (18 patients) was 17.9 months (range, 4.1-39.9). At the time of this analysis, thirteen patients had radiologically-evident tumor progression or recurrence, including 11 cases of local failure and two cases of recurrence out of the radiation field. Four patients died of disease progression, and another patient died from aspiration pneumonia and was excluded from the tumor-related survival analysis.

Table 2 Dose cohorts: carbon-ion radiotherapy (CIRT) boost

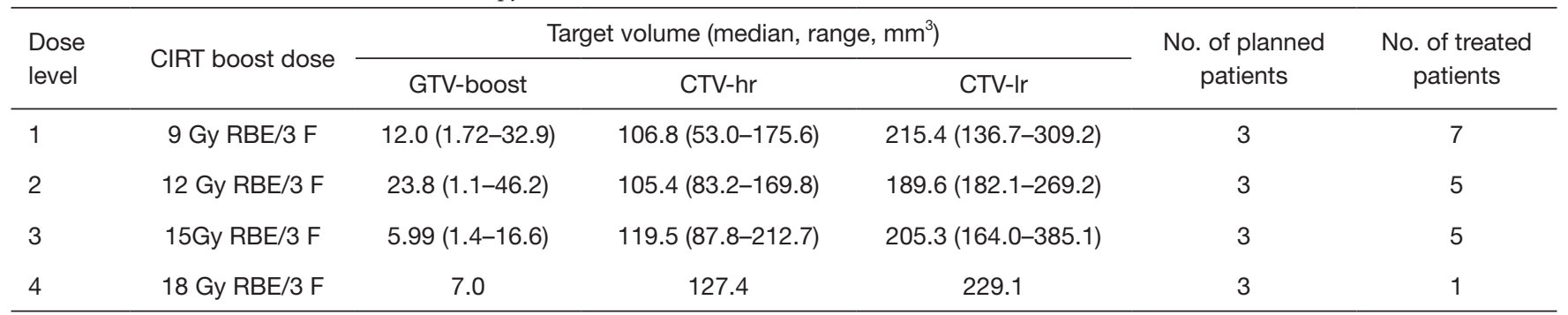

CTV-hr, clinical target volume for high risk; CTV-Ir, clinical target for low risk; GTV-boost, gross tumor volume for boost; RBE, relative biological effectiveness. 
Table 3 Acute and late toxicities

\begin{tabular}{|c|c|c|c|c|c|}
\hline Toxicities type & $\begin{array}{c}\text { Dose level (CIRT } \\
\text { boost) }\end{array}$ & $\begin{array}{l}\text { No. of } \\
\text { cases }\end{array}$ & \multicolumn{3}{|c|}{ Toxicities score } \\
\hline \multirow[t]{2}{*}{ Acute toxicities } & 9 Gy RBE/3 & 4 & Dermatitis, $n=1$; alopecia, $n=4$ & - & - \\
\hline & 12 Gy RBE/3 & 5 & $\begin{array}{l}\text { Dermatitis, } n=1 \text {; alopecia, } n=5 \text {; } \\
\text { epilepsy, } n=1\end{array}$ & - & - \\
\hline \multirow[t]{3}{*}{ Late toxicities } & 9 Gy RBE/3 & 5 & $\begin{array}{l}\text { Radio-necrosis, } n=3 \text {; memory loss, } \\
\qquad n=1 \text {; dizziness, } n=1\end{array}$ & Epilepsy, $n=1$ & - \\
\hline & 12 Gy RBE/3 & 4 & Memory loss, $n=2 ;$ weakness, $n=1$ & $\begin{array}{l}\text { Radio-necrosis, } n=2 ; \text { memory loss, } \\
\qquad n=3 \text {; weakness, } n=1\end{array}$ & - \\
\hline & 18 Gy RBE/3 & 1 & - & - & Radio-necrosis, $n=1$ \\
\hline
\end{tabular}

CIRT, carbon-ion radiotherapy; RBE, relative biological effectiveness.

The median OS of the entire cohort was not reached at the time of this analysis, and the median PFS was 13.5 months. The 12 - and 18 -month PFS rates were $56.7 \%$ [95\% confidence interval (CI), 32.4-81.0\%] and 31.5\% (95\% CI, 8.8-54.2\%), respectively; and the 12- and 18-month OS were both $81.3 \%$ (95\% CI, 62.1-100\%, Figure 2).

At the time of this analysis, both patients with AA who received a CIRT boost of 9 Gy RBE were alive without evidence of disease progression. For the 16 patients with GBM, the median OS was not reached at the time of this analysis, and the PFS was 12.3 months. The 12 and 18-month PFS rates for GBM patients were 50.6\% (95\% CI, $24.3-76.9 \%$ ) and $21.7 \%$ (95\% CI, $2.1-41.3 \%$ ), respectively, and the OS at 12- and 18-month were $78.6 \%$ (95\% CI, $57.0-100 \%)$ and $65.5 \%$ (95\% CI, 36.1-94.9\%), respectively (Figure 2).

\section{Discussion}

The present phase I study consisting of 18 cases is the first clinical trial to evaluate the MTD of a CIRT boost delivered prior to standard-dose PRT (i.e., 60 Gy RBE in 30 fractions) for newly diagnosed HGG. The trial was designed using the $3 \times 3$ scheme and the dose escalation of the CIRT boost included four planned levels from 9-18 Gy $\mathrm{RBE}$ (in 3 fractions). The trial allowed patients to be treated at one prior dose level during the observation period. With a median follow-up of 17.9 months, only one patient treated with CIRT boost to 18 Gy RBE followed by standard dose PRT developed G3 toxicity (radionecrosis), and the MTD of the CIRT boost was defined at $15 \mathrm{~Gy}$ RBE in 3 fractions. In addition, survival analysis indicated a highly acceptable efficacy: the PFS and OS at 12 months for patients with GBM were $50.6 \%$ and $78.6 \%$, respectively; and those for AA were both $100 \%$.

The results of the current standard tri-modality strategy of treating GBM (maximal resection to feasible extent, photon RT with concurrent TMZ, and adjuvant TMZ) remain discouraging, with an expected median OS of around 15 months (2). Histologically, numerous investigations on altering photon-based RT techniques have repeatedly demonstrated failure of this approach to significantly improve the efficacy of GBM treatment (5-8). One of the main factors contributing to the resistance of GBM tumor cells to photon beam is hypoxia; the hallmark characteristic of GBM. Hypoxia has been shown to generally increase the invasiveness and proliferation of GBM tumor cells (20-22). Furthermore, with regards to anti-tumor response, hypoxia produces limitations on the effectiveness of photon radiation and TMZ (23-25), both of which rely on oxygen levels to cause indirect DNA damage and kill tumor cells. Though preclinical data indicated that bevacizumab, the most commonly used antiangiogenic agent in GBM, could lead to transient improvement of intratumoral oxygenation (26), 
A

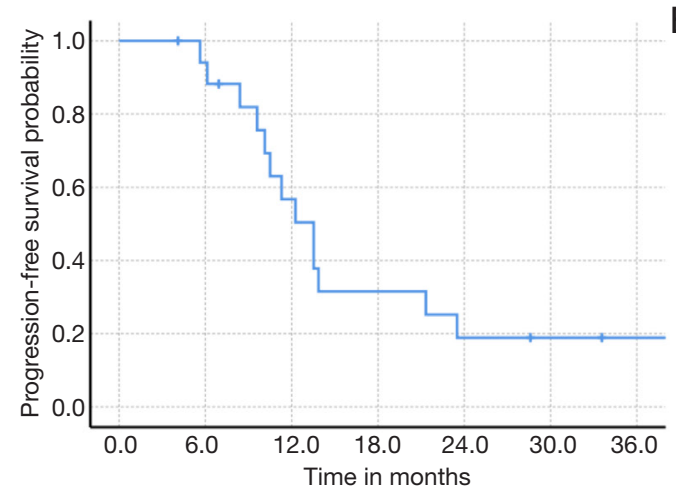

C

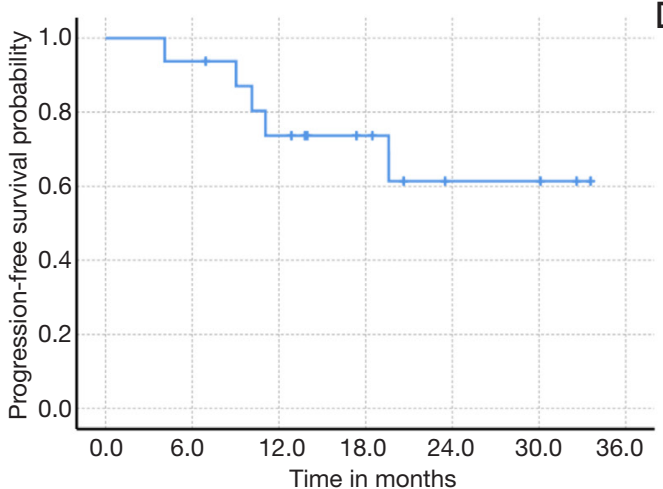

B

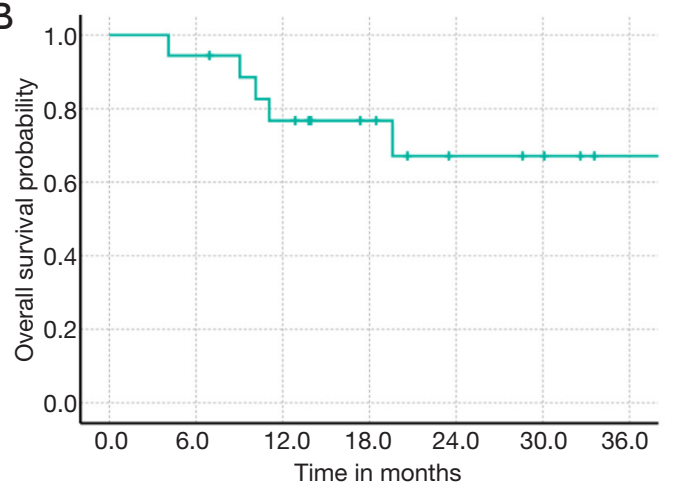

D

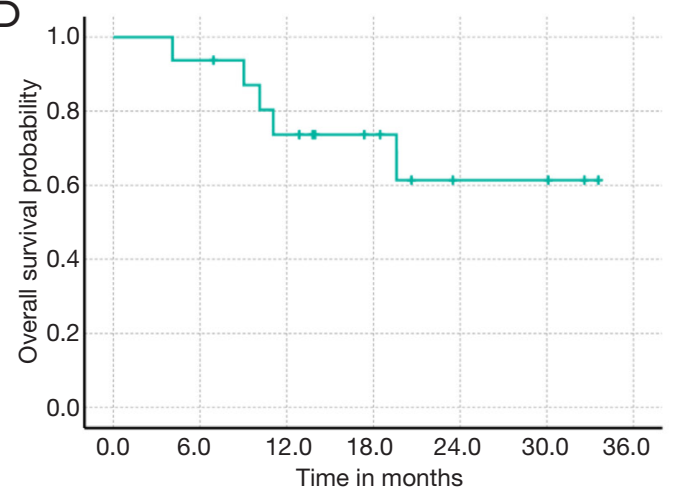

Figure 2 The PFS and OS curves. (A) PFS curve of the entire cohort; (B) OS curve of the entire cohort; (C) PFS curve of GBM patients; (D) OS curve of GBM patients. PFS, progression-free survival; OS, overall survival; GBM, glioblastoma.

numerous clinical studies have shown that the addition of bevacizumab to photon radiotherapy and TMZ failed to improve the overall survival of newly diagnosed GBM (27).

Charged particle beams, such as proton and carbonion, are characterized by a steep dose deposition, known as the Bragg peak, which allows for the surrounding normal critical brain structures to be spared when irradiating brain tumors. As for their biological effects, a proton beam is considered to possess slightly higher biological effectiveness $(\mathrm{RBE}=1.1-1.2)$ than photon beam $(28,29)$. In contrast, a carbon-ion beam, with a much higher RBE value of 3-5 for GBM tumor cells, inflicts more DNA double-strand breaks even under hypoxic conditions (30-34). In addition to direct anti-tumor capability, carbon-ion radiation can also produce a different mechanism of cell death with greater mitotic cell death, autophagy, and apoptosis compared to photon beam radiation, and thereby foster a stronger anti-tumor response (35-38). Thus, PBRT appears to be suitable and promising for the treatment of GBM.

Two previous studies $(10,39)$ have reported the utility of PBRT as a boost in treating HGG. In a phase II trial conducted by the Massachusetts General Hospital (MGH),
23 GBM patients were treated with surgery followed by photon radiation plus PRT boost or PRT up to 90 Gy RBE. The treatment efficacy was encouraging: MST was extended to 20 months; most of the tumor relapse occurred in the areas of dose $\leq 70$ Gy RBE, and only one in a 90 Gy RBE-volume (39). Another retrospective study was jointly reported by the Heidelberg Ion Therapy Center (HIT) of Germany and the National Institute of Radiological Sciences (NIRS) of Japan (10). In this study, 48 patients with HGG (32 GBM and $16 \mathrm{AA}$ ) who received a CIRT boost after photon radiation without chemotherapy were compared with patients treated by photon radiation with and without TMZ. The MSTs were 18 months for GBM patients who received a CIRT boost after photon radiation without chemotherapy, 14 months for patients who received photon radiotherapy with TMZ, and 9 months for patients who received photon radiotherapy without TMZ. The combined information provided in these two studies suggests the hypothesis that dose escalation with PBRT might be necessary if the surrounding crucial brain structures can be spared from high-dose radiation.

Currently, there are two ongoing randomized phase II 
trials (NCT02179086 and NCT01854554) that compare the outcomes of PRT versus photon-based intensity modulated radiotherapy (IMRT) in the treatment of GBM. The NRGBN001 study (NCT02179086) primarily aims to compare the OS of patients treated with dose-escalated photonbased IMRT or PRT versus standard dose 3D-conformal radiation or IMRT (40). The other trial (NCT01854554) is being conducted by MD Anderson Cancer Hospital with the main objective of evaluating the time to cognitive failure between patients treated with PRT versus photon-based IMRT at standard dose/fractionation (41). Both trials hypothesized that more precise or higher dose radiation using proton beam might improve either the disease control or toxicity profile.

As with our phase I trial, PRT was applied at the same dose to the standard photon radiotherapy, which was expected to provide similar or slightly improved biological efficacy, but reduced risk of toxicities due to a lower integral dose to critical brain structures. The CIRT boost was innovatively administered prior to the initiation of PRT. The special timing of the CIRT boost is based on the following theoretical advantages from the unique characteristics of carbon-ion: (I) overcoming hypoxia: the tumor cells' hypoxia level presents their greatest extent in the postoperative setting, thus CIRT with more efficient tumor-killing should be used since its biological impact will not be hindered by hypoxia; (II) targeting glioma stem cells in the early course of treatment: this strategy allows the radiation-naïve tumor stem cells to be irradiated by large-fraction doses of high-LET beam towards a more robust initial cell-killing effect; and (III) altering the immunogenicity within the tumor microenvironment: CIRT is anticipated to shift the common pro-tumor immunity in GBM towards an anti-tumor immunity at the beginning of the radiation-course in the absence of immunosuppressive effect of TMZ and low-LET beam irradiation. Another additional advantage is treatment compliance; patients are more likely to complete the course of CIRT boost if it is given first, while toxicities induced by the 30 -fraction PRT with concurrent TMZ could potentially dissuade the patient from continuing treatment.

Despite the favorable outcomes observed in this phase 1 trial in terms of both survival and adverse effects, further study is needed to understand the role of PBRT in the treatment of HGG. A higher dose of PBRT is unlikely to completely prevent recurrence, even in the high-dose irradiation area. Similar to the most common failure pattern of photon radiotherapy for treating GBM (2), 84.6\%
$(11 / 13)$ of the progression in this study occurred within the PBRT field. In addition, it is debatable whether the 6-month observation interval between the two dose levels is sufficient for the accurate identification of radiationinduced late toxicities. Indeed, the late side effects of CIRT on normal brain tissue are largely unknown at this time. However, considering the limited survival of patients with HGG, and especially considering that the MST of GBM patients treated with current standard treatments is around 15 months (2), we believe that our follow-up time of 17.9 months was sufficient for determining the MTD of the CIRT boost in the setting of a phase I trial. As such, a randomized phase III trial to compare the standard PRT (60 Gy RBE/30 fractions) with an identical PRT regimen plus the addition of a CIRT boost (15 Gy RBE/3 fractions) for GBM patients with residual lesion after resection (16) will soon be conducted.

\section{Conclusions}

PBRT consisting of a CIRT boost of 15 Gy RBE (in 3 fractions) following standard dose PRT of 60 Gy RBE in 30 fractions, used in conjunction with temozolomide, is safe and potentially effective for patients with HGG. The phase III part of our trial will further evaluate the efficacy of the CIRT boost to the MTD (i.e., 15 Gy RBE delivered in 3 fractions) followed by standard dose PRT as compared to PRT alone (16).

\section{Acknowledgments}

Funding: This work was supported by the National Key Research and Development Program of China (Project Nos. 2017YFC0108603 and 2018YFC0115700); the Shanghai Academic/Technology Research Leader Program (Project Nos. 19XD1432900 and 18XD1423000); the Science and Technology Commission of Shanghai Municipality (Project No. 19411951000); the Science and Technology Development Fund of Shanghai Pudong New Area (Project Nos. PKJ2018-Y51, PKJ2017-Y54, PKJ2017-Y50 and No. PKJ2017-Y49); and the Shanghai Municipal Health Commission Special Clinical Research Project (Project No. 20204Y0281).

\section{Footnote}

Reporting Checklist: The authors have completed the TREND reporting checklist. Available at https://atm. 
amegroups.com/article/view/10.21037/atm-20-7750/rc

Data Sharing Statement: Available at https://atm.amegroups. com/article/view/10.21037/atm-20-7750/dss

Conflicts of Interest: All authors have completed the ICMJE uniform disclosure form (available at https://atm.amegroups. com/article/view/10.21037/atm-20-7750/coif). The authors have no conflicts of interest to declare.

Ethical Statement: The authors are accountable for all aspects of the work in ensuring that questions related to the accuracy or integrity of any part of the work are appropriately investigated and resolved. The study was conducted in accordance with the Declaration of Helsinki (as revised in 2013). The study was approved by the ethics committee at Shanghai Proton and Heavy Ion Center (approval number: 1611-12-03-1704A). Written informed consent was obtained from all patients.

Open Access Statement: This is an Open Access article distributed in accordance with the Creative Commons Attribution-NonCommercial-NoDerivs 4.0 International License (CC BY-NC-ND 4.0), which permits the noncommercial replication and distribution of the article with the strict proviso that no changes or edits are made and the original work is properly cited (including links to both the formal publication through the relevant DOI and the license). See: https://creativecommons.org/licenses/by-nc-nd/4.0/.

\section{References}

1. Louis DN, Perry A, Reifenberger G, et al. The 2016 World Health Organization Classification of Tumors of the Central Nervous System: a summary. Acta Neuropathol 2016;131:803-20.

2. Stupp R, Mason WP, van den Bent MJ, et al. Radiotherapy plus concomitant and adjuvant temozolomide for glioblastoma. N Engl J Med 2005;352:987-96.

3. Dong X, Noorbakhsh A, Hirshman BR, et al. Survival trends of grade I, II, and III astrocytoma patients and associated clinical practice patterns between 1999 and 2010: A SEER-based analysis. Neurooncol Pract 2016;3:29-38.

4. Rajaratnam V, Islam MM, Yang M, et al. Glioblastoma: Pathogenesis and Current Status of Chemotherapy and Other Novel Treatments. Cancers (Basel) 2020;12:937.

5. Khan L, Soliman H, Sahgal A, et al. External beam radiation dose escalation for high grade glioma. Cochrane Database Syst Rev 2020;5:CD011475.

6. Cardinale R, Won M, Choucair A, et al. A phase II trial of accelerated radiotherapy using weekly stereotactic conformal boost for supratentorial glioblastoma multiforme: RTOG 0023. Int J Radiat Oncol Biol Phys 2006;65:1422-8.

7. Biswas T, Okunieff P, Schell MC, et al. Stereotactic radiosurgery for glioblastoma: retrospective analysis. Radiat Oncol 2009;4:11.

8. Tsien CI, Brown D, Normolle D, et al. Concurrent temozolomide and dose-escalated intensity-modulated radiation therapy in newly diagnosed glioblastoma. Clin Cancer Res 2012;18:273-9.

9. Schlaich F, Brons S, Haberer T, et al. Comparison of the effects of photon versus carbon ion irradiation when combined with chemotherapy in vitro. Radiat Oncol 2013;8:260.

10. Combs SE, Bruckner T, Mizoe JE, et al. Comparison of carbon ion radiotherapy to photon radiation alone or in combination with temozolomide in patients with high-grade gliomas: explorative hypothesis-generating retrospective analysis. Radiother Oncol 2013;108:132-5.

11. Combs SE, Ellerbrock M, Haberer T, et al. Heidelberg Ion Therapy Center (HIT): Initial clinical experience in the first 80 patients. Acta Oncol 2010;49:1132-40.

12. Chiblak S, Tang Z, Campos B, et al. Radiosensitivity of Patient-Derived Glioma Stem Cell 3-Dimensional Cultures to Photon, Proton, and Carbon Irradiation. Int J Radiat Oncol Biol Phys 2016;95:112-9.

13. Combs SE, Nikoghosyan A, Jaekel O, et al. Carbon ion radiotherapy for pediatric patients and young adults treated for tumors of the skull base. Cancer 2009;115:1348-55.

14. Schulz-Ertner D, Nikoghosyan A, Hof H, et al. Carbon ion radiotherapy of skull base chondrosarcomas. Int J Radiat Oncol Biol Phys 2007;67:171-7.

15. Koto M, Demizu Y, Saitoh JI, et al. Multicenter Study of Carbon-Ion Radiation Therapy for Mucosal Melanoma of the Head and Neck: Subanalysis of the Japan Carbon-Ion Radiation Oncology Study Group (J-CROS) Study (1402 HN). Int J Radiat Oncol Biol Phys 2017;97:1054-60.

16. Kong L, Gao J, Hu J, et al. Carbon ion radiotherapy boost in the treatment of glioblastoma: a randomized phase I/III clinical trial. Cancer Commun (Lond) 2019;39:5.

17. Emami B, Lyman J, Brown A, et al. Tolerance of normal tissue to therapeutic irradiation. Int J Radiat Oncol Biol Phys 1991;21:109-22.

18. Wen PY, Macdonald DR, Reardon DA, et al. Updated 
response assessment criteria for high-grade gliomas: response assessment in neuro-oncology working group. J Clin Oncol 2010;28:1963-72.

19. Ellingson BM, Wen PY, Cloughesy TF. Modified Criteria for Radiographic Response Assessment in Glioblastoma Clinical Trials. Neurotherapeutics 2017;14:307-20.

20. da Silva R, Uno M, Marie SK, et al. LOX expression and functional analysis in astrocytomas and impact of IDH1 mutation. PLoS One 2015;10:e0119781.

21. Scribner E, Saut O, Province P, et al. Effects of antiangiogenesis on glioblastoma growth and migration: model to clinical predictions. PLoS One 2014;9:e115018.

22. Wolf A, Agnihotri S, Micallef J, et al. Hexokinase 2 is a key mediator of aerobic glycolysis and promotes tumor growth in human glioblastoma multiforme. J Exp Med 2011;208:313-26.

23. Lo Dico A, Martelli C, Diceglie C, et al. HypoxiaInducible Factor-1alpha Activity as a Switch for Glioblastoma Responsiveness to Temozolomide. Front Oncol 2018;8:249.

24. Chen WL, Wang CC, Lin YJ, et al. Cycling hypoxia induces chemoresistance through the activation of reactive oxygen species-mediated B-cell lymphoma extralong pathway in glioblastoma multiforme. J Transl Med 2015;13:389.

25. Shen H, Cook K, Gee HE, et al. Hypoxia, metabolism, and the circadian clock: new links to overcome radiation resistance in high-grade gliomas. J Exp Clin Cancer Res 2020;39:129.

26. Nanegrungsunk D, Onchan W, Chattipakorn N, et al. Current evidence of temozolomide and bevacizumab in treatment of gliomas. Neurol Res 2015;37:167-83.

27. Kaka N, Hafazalla K, Samawi H, et al. ProgressionFree but No Overall Survival Benefit for Adult Patients with Bevacizumab Therapy for the Treatment of Newly Diagnosed Glioblastoma: A Systematic Review and MetaAnalysis. Cancers (Basel) 2019;11:1723.

28. Rimpl GR, Schmid E, Braselmann H, et al. Chromosome aberrations induced in human lymphocytes by $16.5 \mathrm{MeV}$ protons. Int J Radiat Biol 1990;58:999-1007.

29. Takei H, Inaniwa T. Effect of Irradiation Time on Biological Effectiveness and Tumor Control Probability in Proton Therapy. Int J Radiat Oncol Biol Phys 2019;105:222-9.

30. Heilmann J, Taucher-Scholz G, Haberer T, et al. Measurement of intracellular dna double-strand break induction and rejoining along the track of carbon and neon particle beams in water. Int J Radiat Oncol Biol Phys 1996;34:599-608.

31. Murakami M, Eguchi-Kasai K, Sato K, et al. Differences in heavy-ion-induced DNA double-strand breaks in a mouse DNA repair-deficient mutant cell line (SL3-147) before and after chromatin proteolysis. J Radiat Res 1995;36:258-64.

32. Tsuboi K, Moritake T, Tsuchida Y, et al. Cell cycle checkpoint and apoptosis induction in glioblastoma cells and fibroblasts irradiated with carbon beam. J Radiat Res 2007;48:317-25.

33. Combs SE, Zipp L, Rieken S, et al. In vitro evaluation of photon and carbon ion radiotherapy in combination with chemotherapy in glioblastoma cells. Radiat Oncol 2012;7:9.

34. Combs SE, Bohl J, Elsasser T, et al. Radiobiological evaluation and correlation with the local effect model (LEM) of carbon ion radiation therapy and temozolomide in glioblastoma cell lines. Int J Radiat Biol 2009;85:126-37.

35. Ferrandon S, Magne N, Battiston-Montagne P, et al. Cellular and molecular portrait of eleven human glioblastoma cell lines under photon and carbon ion irradiation. Cancer Lett 2015;360:10-6.

36. Maalouf M, Alphonse G, Colliaux A, et al. Different mechanisms of cell death in radiosensitive and radioresistant p53 mutated head and neck squamous cell carcinoma cell lines exposed to carbon ions and $\mathrm{x}$-rays. Int J Radiat Oncol Biol Phys 2009;74:200-9.

37. Shimada M, Hirayama R, Komatsu K. High LET radiation amplifies centrosome overduplication through a pathway of gamma-tubulin monoubiquitination. Int J Radiat Oncol Biol Phys 2013;86:358-65.

38. Yoshimoto Y, Oike T, Okonogi N, et al. Carbon-ion beams induce production of an immune mediator protein, high mobility group box 1 , at levels comparable with X-ray irradiation. J Radiat Res 2015;56:509-14.

39. Fitzek MM, Thornton AF, Rabinov JD, et al. Accelerated fractionated proton/photon irradiation to 90 cobalt gray equivalent for glioblastoma multiforme: results of a phase II prospective trial. J Neurosurg 1999;91:251-60.

40. Mehta M. Randomized phase II trial of hypofractionated dose-escalated photon IMRT or proton beam therapy versus conventional photon irradiation with concomitant and adjuvant temozolomide in patients with newly diagnosed glioblastoma. Available online: https:// clinicaltrials.gov/ct2/show/NCT02179086. Accessed October 102020. 
41. Chung C. A Prospective Phase II Randomized trial to compare intensity modulated proton radiotherapy (IMPT) vs. intensity modulated radiotherapy (IMRT) for newly diagnosed glioblastoma (WHO Grade IV). Available

Cite this article as: Qiu X, Gao J, Yang J, Hu J, Hu W, Huang Q, Kong L, Lu JJ. Carbon-ion radiotherapy boost with standard dose proton radiation for incompleteresected high-grade glioma: a phase 1 study. Ann Transl Med 2022;10(22):1193. doi:10.21037/atm-20-7750 online: https://clinicaltrials.gov/ct2/show/NCT01854554. Accessed October 102020.

(English Language Editor: A. Kassem) 